\title{
Crystal structure of the translation recovery factor Trf from Sulfolobus solfataricus
}

\author{
Marco Kaiser ${ }^{1}$, Jan Philip Wurm ${ }^{1, \dagger}$, Birgit Märtens ${ }^{2}$, Udo Bläsi ${ }^{2}$, Denys Pogoryelov ${ }^{3}$ and \\ Jens Wöhnert ${ }^{1}$ \\ 1 Institute of Molecular Biosciences and Center for Biomolecular Magnetic Resonance (BMRZ), Goethe-University Frankfurt, Germany \\ 2 Department of Microbiology, Immunobiology and Genetics, Max F. Perutz Laboratories, Center of Molecular Biology, \\ University of Vienna, Austria \\ 3 Institute of Biochemistry, Goethe-University Frankfurt, Germany
}

\section{Keywords \\ DUF35; ribosome; Sulfolobus solfataricus: translation initiation; translation recovery factor Trf \\ Correspondence \\ J. Wöhnert, Institute of Molecular Biosciences, Goethe-University Frankfurt, Max-von-Laue-Str. 9, 60438 Frankfurt, Germany \\ Fax: +49-69-79829527 \\ Tel: +49-69-79829785 \\ E-mail: woehnert@bio.uni-frankfurt.de \\ †Present Address \\ Institute of Biophysics and Physical Biochemistry, University of Regensburg, Germany}

(Received 13 September 2019, revised 25 November 2019, accepted 3 December 2019)

doi:10.1002/2211-5463.12772
During translation initiation, the heterotrimeric archaeal translation initiation factor 2 (aIF2) recruits the initiator $\mathrm{tRNA}_{\mathrm{i}}$ to the small ribosomal subunit. In the stationary growth phase and/or during nutrient stress, Sulfolobus solfataricus aIF2 has a second function: It protects leaderless mRNAs against degradation by binding to their $5^{\prime}$-ends. The $S$. solfataricus protein Sso2509 is a translation recovery factor (Trf) that interacts with aIF2 and is responsible for the release of aIF2 from bound mRNAs, thereby enabling translation re-initiation. It is a member of the domain of unknown function 35 (DUF35) protein family and is conserved in Sulfolobales as well as in other archaea. Here, we present the X-ray structure of $S$. solfataricus $\operatorname{Trf}$ solved to a resolution of $1.65 \AA$. Trf is composed of an $\mathrm{N}$-terminal rubredoxin-like domain containing a bound zinc ion and a C-terminal oligosaccharide/oligonucleotide binding fold domain. The Trf structure reveals putative mRNA binding sites in both domains. Surprisingly, the Trf protein is structurally but not sequentially very similar to proteins linked to acyl-CoA utilization-for example, the Sso2064 protein from $S$. solfataricus - as well as to scaffold proteins found in the acetoacetyl-CoA thiolase/high-mobility group-CoA synthase complex of the archaeon Methanothermococcus thermolithotrophicus and in a steroid sidechain-cleaving aldolase complex from the bacterium Thermomonospora curvata. This suggests that members of the DUF35 protein family are able to act as scaffolding and binding proteins in a wide variety of biological processes.
Sulfolobus solfataricus is a thermoacidophilic archaeon originally isolated from hot volcanic springs. It grows optimally at $350 \mathrm{~K}$ and in acidic environments with a $\mathrm{pH}$ between 2 and 4 [1]. Fundamental features of translation initiation are conserved between archaea such as $S$. solfataricus and eukaryotes [2]. The heterotrimeric translation initiation factor $\operatorname{IF} 2(\alpha, \beta$, and $\gamma)$ is required for binding of the methionine-loaded initiator tRNA (tRNA ${ }_{\mathrm{i}}$ ) to the small ribosomal subunit as well as the proper positioning of tRNA $\mathrm{A}_{\mathrm{i}}$ in the ribosomal P-site in archaea and in eukaryotes. However, crenarchaeal archaeal initiation factor 2 (aIF2) lacks the $\mathrm{tRNA}_{\mathrm{i}}$ shuttle function of eukaryotic initiation factor $2[3,4]$. Interestingly, in S. solfataricus the aIF $2 \gamma$ subunit has an additional function during the stationary growth phase and/or during nutrient

\section{Abbreviations}

alF2, archaeal initiation factor 2; DUF, domain of unknown function; EMSA, electrophoretic mobility shift assay; OB-fold, oligosaccharide/ oligonucleotide binding fold; PDB, Protein Data Bank; Trf, translation recovery factor. 
starvation. Under these conditions, it protects mRNAs against exonuclease-mediated $5^{\prime}-3^{\prime}$ decay via binding to their triphosphorylated $5^{\prime}$-ends $[5,6]$. To reinitiate translation after nutrient replenishment, aIF $2 \gamma$ must be displaced from mRNAs. The small protein translation recovery factor (Trf) was identified as the factor responsible for releasing aIF $2 \gamma$ from bound mRNAs, thereby enabling re-initiation of translation after relief of nutrient stress [5].

A sequence alignment of Trf homologs from different Sulfolobus species revealed two conserved regions predicted to be similar to an N-terminal rubredoxinlike and a C-terminal oligosaccharide/oligonucleotide binding fold (OB-fold) domain [5]. Here, we report the crystal structure of the 124-amino acid (aa) S. solfataricus Trf protein (Sso2509) at a resolution of $1.65 \AA$. Interestingly, the structure but not the sequence of $\operatorname{Trf}$ is similar to other proteins of the domain of unknown function 35 (DUF35) family [Protein Data Bank (pdb): pdb 3irb, 6et9 chain E, 6ok1 chain B, 5m3k chain $\mathrm{B}$ ] that have been described to bind directly or indirectly to acyl-CoA $[7,8]$ or act as scaffolding components of acyl-CoA-dependent enzyme complexes $[8,9]$ as well as a CoA-independent bacterial acylase [10]. This suggests a general role of DUF35 family proteins as scaffolding or binding proteins in a variety of biological processes.

\section{Materials and methods}

A codon-optimized gene for production of $\operatorname{Trf}$ in Escherichia coli was obtained commercially from GenScript in a pUC57 plasmid vector. The gene encoded the fulllength native Trf protein sequence from $S$. solfataricus. The coding region was subcloned into a pET11a expression plasmid using the NdeI and BamHI restriction sites.

Escherichia coli BL21 (DE3) cells were transformed with this plasmid, and cells were grown at $37{ }^{\circ} \mathrm{C}$ in Luria broth medium supplemented with $100 \mathrm{mg} \cdot \mathrm{mL}^{-1}$ ampicillin until an optical density $\left(\mathrm{OD}_{600}\right)$ of 0.8 was reached. Trf synthesis was induced by adding $1 \mathrm{~mm}$ isopropyl $\beta$-D-1-thio-galactopyranoside at $20^{\circ} \mathrm{C}$. Sixteen hours after induction, the cells from a $1 \mathrm{~L}$ culture were harvested by centrifugation at $5000 \mathrm{~g}$ $\left(10 \mathrm{~min}, 4{ }^{\circ} \mathrm{C}\right.$ ) and disrupted by sonication in $20 \mathrm{~mL}$ lysis buffer (50 mm BisTris pH 5.8, $200 \mathrm{~mm} \mathrm{NaCl,} 10 \mathrm{~mm}$ B-mercaptoethanol, $200 \mathrm{~mm}$ imidazole) on ice. After cell lysis, a heat denaturation step was performed at $65^{\circ} \mathrm{C}$ for $15 \mathrm{~min}$. Subsequently, the solution was centrifuged at $7000 \mathrm{~g}$ $\left(20 \mathrm{~min}, 4{ }^{\circ} \mathrm{C}\right)$. The supernatant containing $\operatorname{Trf}$ was diluted with dilution buffer (50 mM BisTris $\mathrm{pH} 5.8,10 \mathrm{~mm}$ B-mercaptoethanol, $200 \mathrm{~mm}$ imidazole) to $150 \mathrm{~mL}$ and loaded onto a SP-Sepharose column (GE Healthcare, Solingen, Germany, HiPrepTM SP FF 16/60). Trf was eluted with a buffer containing $1 \mathrm{M} \mathrm{NaCl}$ in a linear gradient. Fractions containing Trf were pooled, concentrated to $4 \mathrm{~mL}$, and applied to a gel filtration column (Superdex 200 HR 26/60; GE Healthcare) pre-equilibrated with gel filtration buffer (50 mм BisTris pH 6.5, $50 \mathrm{~mm} \mathrm{NaCl}, 5 \mathrm{~mm}$ ß-mercapthoethanol, $200 \mathrm{~mm}$ imidazole). Fractions containing Trf were pooled and concentrated to a final protein concentration of $175 \mu \mathrm{M}$. Protein purity was assessed by SDS/PAGE. MALDI mass spectrometry yielded a molecular weight of $14.78 \mathrm{kDa}$ (calculated $14.78 \mathrm{kDa}$ ).

Crystallization of $\operatorname{Trf}$ was achieved by slowly removing imidazole from the buffer during a two-step dialysis of a gel filtration sample $(4 \mathrm{~mL} / 175 \mu \mathrm{M})$ of $\operatorname{Trf}$ against $1 \mathrm{~L}$ of an imidazole-free dialysis buffer (50 mм BisTris pH 6.5, $50 \mathrm{~mm}$ $\mathrm{NaCl}, 5 \mathrm{~mm} \mathrm{~B}$-mercapthoethanol) at $4{ }^{\circ} \mathrm{C}$ in a dialysis volume of $500 \mathrm{~mL}$. After $24 \mathrm{~h}$, the buffer was exchanged, and dialysis was continued for additional $72 \mathrm{~h}$. After 4 days of dialysis, crystals appeared in the dialysis chamber (Slide-ALyzer $^{\mathrm{TM}}$, Thermo Fisher, Waltham, MA, USA, $3.5 \mathrm{kDa}$ molecular weight cutoff). A microscopic analysis revealed rod-like crystals being UV-active. These crystals were used for structure determination. The crystals were cryoprotected by adding 33\% PEG-400 to the crystallization solution.

$\mathrm{X}$-ray diffraction data were collected on the beamline station PXIII of the Swiss Light Source (Paul Scherrer Institut, Villigen, Switzerland). All diffraction data were obtained from a single crystal and processed with the XDS software package [11]. The positions of anomalous scatterers were determined using SHELXD [12]. The initial phases were obtained by SHELXE $[13,14]$, and the initial model was built automatically by RESOLVE [15]. The initial model was extended by iterative rounds of model building with COOT [16] into the $2 \mathrm{~F}_{\mathrm{o}}-\mathrm{F}_{\mathrm{c}}$ electron density map and refined using the PHENIX software package [17]. The Ramachandran plots of the final structure showed no outlying residues, as assessed by model validation with the program MOLPROBITY [18]. The graphical representations were generated using CHIMERA [19]. Proteins that are structurally similar to Trf were identified using the DALI server [20] and PDBeFOLD [21]. $\mathrm{C} \alpha$ RMSDs and $Z$-scores were calculated using the pairwise structure comparison option available at the DALI server.

Fluorescence anisotropy-based RNA binding assays were performed at $25^{\circ} \mathrm{C}$ using a Fluorolog 3 spectrometer (Horiba, Bensheim, Germany). Excitation and emission wavelengths were set to 492 and $521 \mathrm{~nm}$. 5'-fluorescein-labeled RNAs were obtained commercially (Dharmacon, Lafayette, CO, USA) and deprotected according to the instructions of the manufacturer. Experiments were performed in a buffer containing $50 \mathrm{~mm}$ BisTris, $\mathrm{pH}$ 6.5, and $100 \mathrm{~mm} \mathrm{NaCl}$. A $\mathrm{NaCl}$ concentration of $100 \mathrm{~mm}$ in the buffer was necessary to prevent precipitation of the protein. The concentrations of the $5^{\prime}$-fluorescein-labeled RNAs were $100 \mathrm{~nm}$. Trf was added stepwise until saturation was reached. Titration experiments were performed in triplicate. Each data point represents the average value with error bars indicating the standard deviation. 
Analytical gel filtration in a buffer containing $50 \mathrm{~mm}$ BisTris, $\mathrm{pH} 6.5$, and $150 \mathrm{~mm} \mathrm{NaCl}$ was performed as described previously [22]. A protein concentration of $50 \mu \mathrm{M}$ was used. For molecular weight comparisons, we used the proteins $P h \mathrm{~S} 11$ and $P h$ Fap 7 as well as the highly stable PhFap7/PhS11 complex with molecular weights of 14.74 , 20.18, and $34.92 \mathrm{kDa}$, respectively [18].

\section{Results and Discussion}

Structural information for the 124 aa protein $\operatorname{Trf}$ responsible for the release of mRNAs bound to aIF $2 \gamma$ during the outgrowth phase in $S$. solfataricus was so far not available [5]. Here, we report the crystal structure of Trf determined from a cuboid needle-like crystal diffracting to a resolution of $1.65 \AA$ (Table 1 ). Previously, a sequence alignment suggested a N-terminal rubredoxin-like domain followed by a C-terminal OB-fold domain [5]. The X-ray structure (Fig. 1A) confirmed that Trf contains two domains, an N-terminal rubredoxin-like domain (aa: 19-51) and a C-terminal OB-fold-like domain (aa: 54-117). Additionally, a short $\alpha$-helix ( $\alpha 1$, aa $3-15)$ precedes the rubredoxin-like domain which is composed of two antiparallel $\beta$ strands $(\beta 1$, aa $19-24 ; \beta 2$, aa 46-51) surrounding a loop region (aa 25-45), which complexes a zinc ion. The zinc ion is coordinated by four cysteine residues (aa 24, 27, 38, and 41) of the loop region (Fig. 1B). The $\beta$-barrel of the OB-fold-like domain is formed by five antiparallel $\beta$-strands ( $\beta 3-\beta 7$, aa $54-65$; $69-78$; $83-$ 88; $99-107$; and $110-117$ ) as is typical for this type of domain [23].

The asymmetric unit contains two molecules (chains A and B; Fig. 1A). For both molecules, the refined model begins with residue 1 and terminates with residue 119. The C-terminal residues His120, Leu121, His122, Asn123, and Phe124 could not be resolved due to insufficient electron density suggestive of a flexible $\mathrm{C}$ terminus. Overall, the structure contains the two protein chains $\mathrm{A}$ and $\mathrm{B}$, two zinc ions, and 257 water molecules. Both monomers of the unit cell are virtually identical with an overall RMSD for C $\alpha$ atoms 1-119 of $0.739 \AA$ (Fig. 1C). However, the dimer interface corresponds to a buried solvent-accessible surface area of only $789 \AA^{2}$ as determined with PDBePISA. This argues against the formation of a stable Trf dimer in solution [24]. The retention volume of $\operatorname{Trf}$ on an analytical gel filtration column suggests that Trf in solution is indeed a monomeric protein (Fig. 1D).

An electrostatic surface representation of $\operatorname{Trf}$ shows a positively charged surface for $\beta$-strands $\beta 3, \beta 4$, and $\beta 5$ which correspond to the first three $\beta$-strands of the OB-fold domain as well as for a part of the zinc
Table 1. Data collection and refinement statistics. Statistics for the highest resolution shell are shown in parentheses.

\begin{tabular}{ll}
\hline Wavelength & 1.0 \\
Resolution range & $37.44-1.65(1.709-1.65)$ \\
Space group & P 212121 \\
Unit cell & 40.29158 .493101 .366909090 \\
Total reflections & $398915(34092)$ \\
Unique reflections & $29528(2817)$ \\
Multiplicity & $13.5(12.1)$ \\
Completeness (\%) & $99.82(98.26)$ \\
Mean I/sigma () & $13.32(1.78)$ \\
Wilson B-factor & 17.80 \\
$R$-merge & $0.1447(1.238)$ \\
$R$-meas & $0.1504(1.292)$ \\
$R$-pim & $0.04051(0.3628)$ \\
CC1/2 & $0.998(0.662)$ \\
CC* & $1.0(0.893)$ \\
Reflections used in refinement & $29528(2817)$ \\
Reflections used for $R$-free & $1476(140)$ \\
$R$-work & $0.1915(0.2793)$ \\
$R$-free & $0.2070(0.3074)$ \\
CC (work) & $0.959(0.823)$ \\
CC (free) & $0.966(0.814)$ \\
Number of nonhydrogen atoms & 2219 \\
Macromolecules & 1964 \\
Ligands & 2 \\
Solvent & 253 \\
Protein residues & 237 \\
RMS (bonds) & 0.012 \\
RMS (angles) & 1.18 \\
Ramachandran favored (\%) & 99.14 \\
Ramachandran allowed (\%) & 0.86 \\
Ramachandran outliers (\%) & 0.00 \\
Rotamer outliers (\%) & 0.00 \\
Clashscore & 3.57 \\
Average B-factor & 25.74 \\
Macromolecules & 24.79 \\
Ligands & 13.77 \\
Solvent & 33.17 \\
\hline & \\
\hline & \\
\hline
\end{tabular}

ribbon motif (Fig. 2A). These two positively charged surface areas are located close to each other and might therefore represent a possible interaction platform for nucleic acids. This would correspond to the RNAbinding site in many other OB-fold proteins where often $\beta$-strands 2 and 3 sometimes $\beta$-strand 1 represent the recognition and binding surface for their nucleic acid ligands $[23,25]$. Two other prominent determinants for RNA binding in OB-folds are the two loops connecting $\beta$-strands $\beta 1$ and $\beta 2\left(\mathrm{~L}_{12}\right)$ as well as $\beta 4$ and $\beta 5\left(\mathrm{~L}_{45}\right)$. In agreement with an RNA binding function for Trf, these loops contain a significant number of basic residues (R65 and $\mathrm{K} 66$ in $\mathrm{L}_{12}$ and $\mathrm{K} 105, \mathrm{~K} 110$, and $\mathrm{K} 111$ in $\mathrm{L}_{45}$ ) augmented by aromatic residues (Y64 and $\mathrm{Y} 70$ in $\mathrm{L}_{12}$ as well as $\mathrm{Y} 112$ in $\beta 4$ ) that could contribute to RNA binding by providing base stacking 

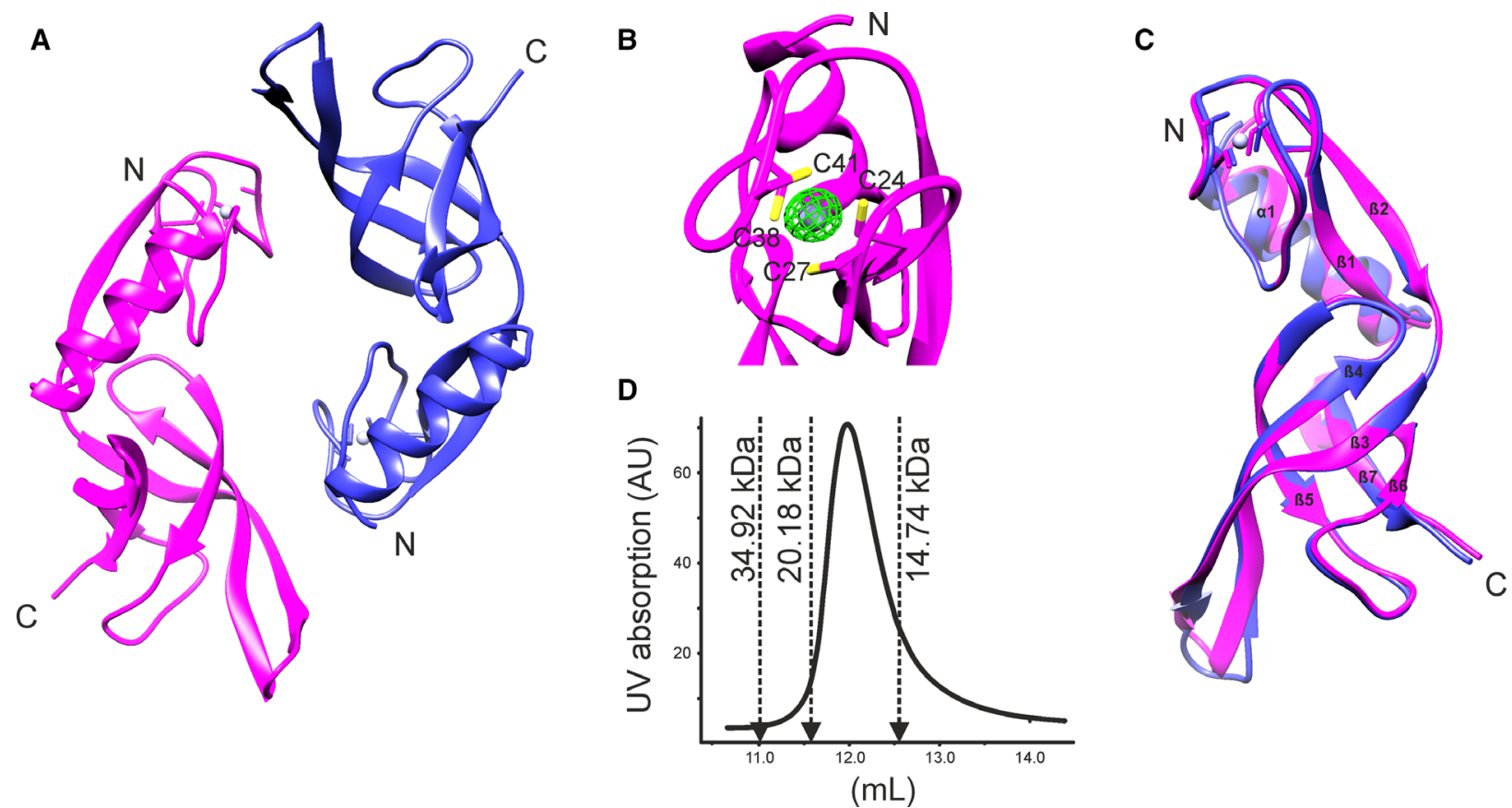

Fig. 1. Structure of Trf. (A) Cartoon representation of the $X$-ray structure for the Trf homodimer found in the crystal. Chain $A$ is shown in magenta, chain B in medium blue, and the bound zinc ion as a blue/gravy sphere. (B) Close-up of the zinc coordination site of Trf. The zinccoordinating cysteine side chains are labeled. The electron density difference map $\left(F_{o}-F_{c}\right)$ for the zinc ion is shown in green at the $3 \sigma$ level. (C) Superimposition of the two monomers of the crystallographic Trf dimer. Secondary structure elements are named and numbered. (D) Analytical gel filtration profile of Trf which shows that Trf is monomeric in solution. Arrows indicate the elution volumes of marker proteins in the same buffer (PhFap7: 20.18 kDa; PhS11: 14.74 kDa; PhFap7/PhS11 complex: 34.92 kDa).

interactions. Furthermore, many OB-fold proteins feature a solvent-exposed hydrophobic residue at the center of strand 3 which is often involved in stacking interactions with nucleic acid bases [23,25]. This residue is also present in $\operatorname{Trf}$ as $\mathrm{Y} 85$.

However, in previous experiments using electrophoretic mobility shift assays (EMSA) no evidence for direct RNA binding by $\operatorname{Trf}$ was found [5]. We tested the binding of Trf to a single-stranded RNA and an RNA that contains a hairpin loop next to single-stranded segments which were $5^{\prime}$-fluorescein-labeled with fluorescence anisotropy measurements. Fluorescence anisotropy measurements are sensitive to binding events over a broader $K_{\mathrm{D}}$ range compared to EMSA experiments. In titration experiments, we found that Trf bound these RNAs with $K_{\mathrm{D}}$ values in the low micromolar range (Fig. 2B). Thus, Trf apparently has a genuine RNA-binding capacity in agreement with its proposed function. However, the additional presence of a structured hairpin loop element in one of the RNAs did not lead to an increased affinity of Trf for this RNA.

To find structurally similar proteins in the PDB, we used the DALI server [20] as well as PDBeFold [21].
Trf has structural homology to four other published structures (PDB 3irb, 6et9, 6ok1, and 5m3k, Fig. 3A). All four proteins also belong to the DUF35 family [710] but apparently have no RNA-related functions. The first example-a protein termed Sso2064-also occurs in S. solfataricus [7]. Functionally, Sso2064 is linked to acetyl-CoA binding related to functions in the biosynthesis of lipids and polyketide antibiotics based on a genome context analysis [7]. Sso2064 also crystallized as a homodimer similar to our observations for Trf. The superimposition of Trf with Sso2064 shows a similar overall fold with a C $\alpha$ RMSD of 2.3 $\AA$ for 108 aligned residues and a DALI $Z$-score of 10.4. The second DUF35 protein was recently identified as a scaffolding subunit in the acetoacetyl-CoA thiolase/high-mobility group-CoA synthase complex of Methanothermococcus thermolithotrophicus (pdb entry 6et9 chain E) which is part of the mevalonate pathway [8]. The DUF35 subunit (chain E) in pdb entry 6et9 aligns to Trf with a C $\alpha$ RMSD of $2.2 \AA$ for 107 residues with a DALI $Z$-score of 12.7. Another example for a DUF35 protein acting as a scaffolding subunit in a CoA-dependent enzyme complex was found in the structure of a steroid side-chain-cleaving aldolase [9] 

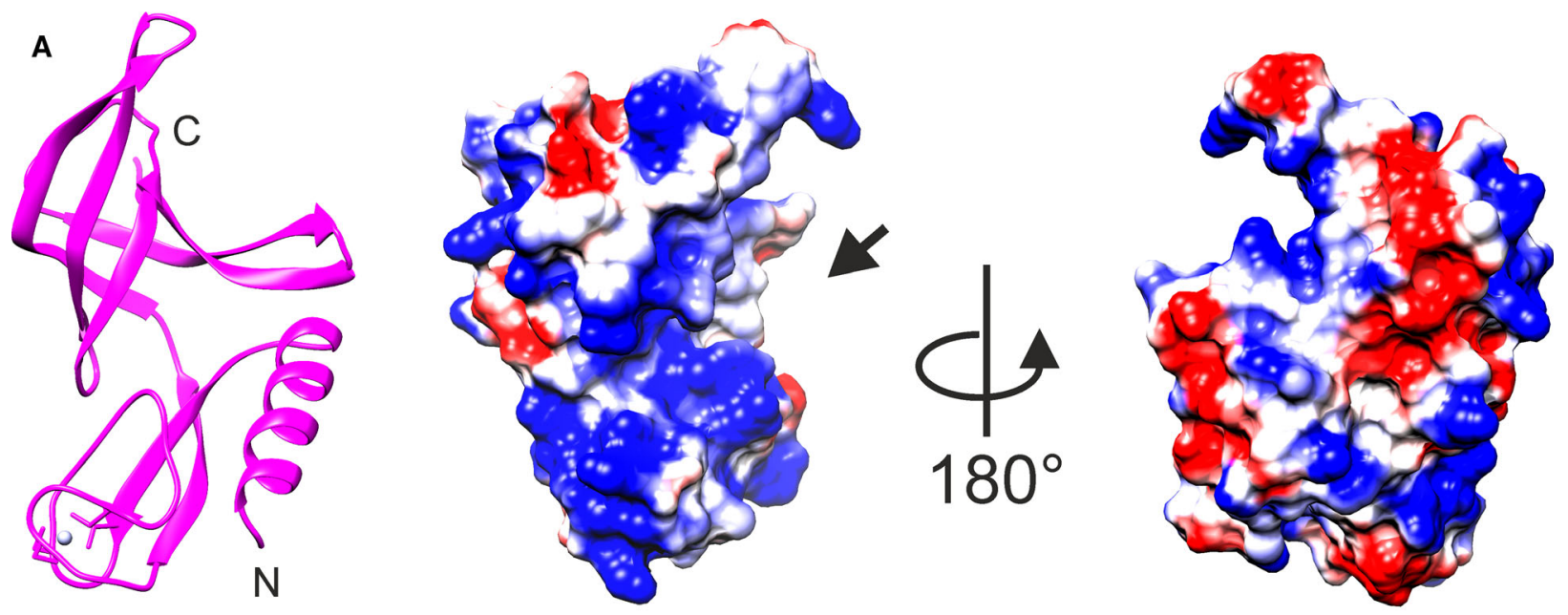

B
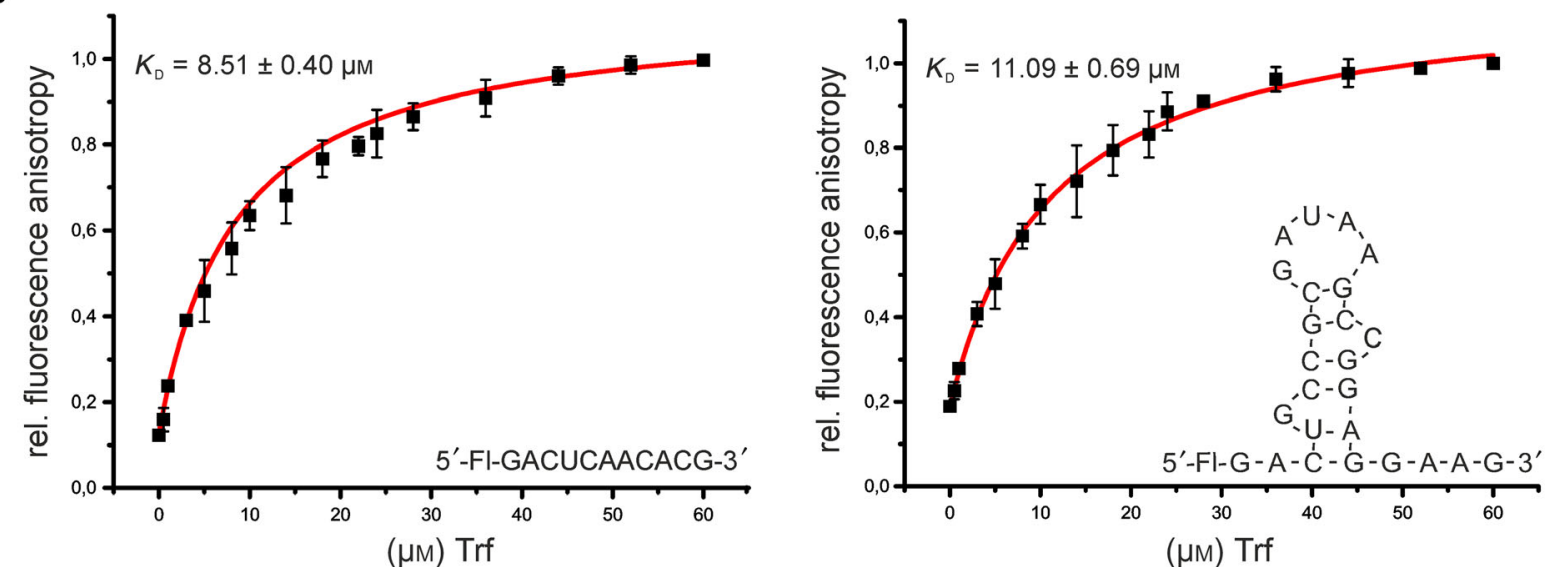

Fig. 2. Electrostatic surface potential and RNA-binding capability of Trf (A) Electrostatic surface potential of Trf. Blue colors correspond to positively charged areas, red colors to negatively charged areas, and white to areas with neutral electrostatic potential. For orientation, a cartoon representation of the structure of the Trf monomer in the same orientation is shown on the left. A positively charged putative RNA binding cleft is indicated by an arrow. (B) Change in fluorescence anisotropy upon titration of Trf to fluorescein-labeled single-stranded and hairpin RNAs. RNA sequences, secondary structures, and the resulting $K_{\mathrm{D}}$ values are depicted in the corresponding figure panels. Titration experiments were performed in triplicate. Each data point represents the average value with error bars indicating the standard deviation.

from the bacterium Thermomonospora curvata (pdb entry $60 \mathrm{k} 1$, chain $\mathrm{B})$. The structure of this protein aligns to Trf with a C $\alpha$ RMSD of $2.4 \AA$ for 116 aligned residues (DALI $Z$-score 15.4). DUF35 protein family members can also occur as a scaffolding subunit in CoA-independent enzymes [10] as illustrated by the structure of a Friedel-Crafts acylase complex from the bacterium Pseudomonas protegens ( $\mathrm{pdb}$ entry $5 \mathrm{~m} 3 \mathrm{k}$, chain B). However, it should be noted that this enzyme complex is apparently structurally and evolutionarily related to CoA-dependent enzymes [10]. The DUF35 subunit of this complex aligns to $\operatorname{Trf}$ with a C $\alpha$ RMSD of $2.5 \AA$ for 110 aligned residues (DALI $Z$-score 12.0). All DUF35 proteins structurally characterized so far bind a zinc ion in equivalent positions. In those cases where the DUF35 homolog was crystallized as a scaffolding protein in the context of larger hetero-oligomeric assemblies (6et9, 6ok1, and $5 \mathrm{~m} 3 \mathrm{k}$ ) which are either dimers of heterodimers or dimers of heterotrimers, the DUF35 subunits are always involved in extensive interactions with the other subunits of the complex and never stabilize these complexes by homotopic interactions with each other.

In comparison with Sso2064, all other structurally characterized DUF35 family members including Trf lack an additional long $\mathrm{N}$-terminal $\alpha$-Helix (Fig. 3B). Despite the high degree of structural similarity between all proteins, the level of sequence identity 

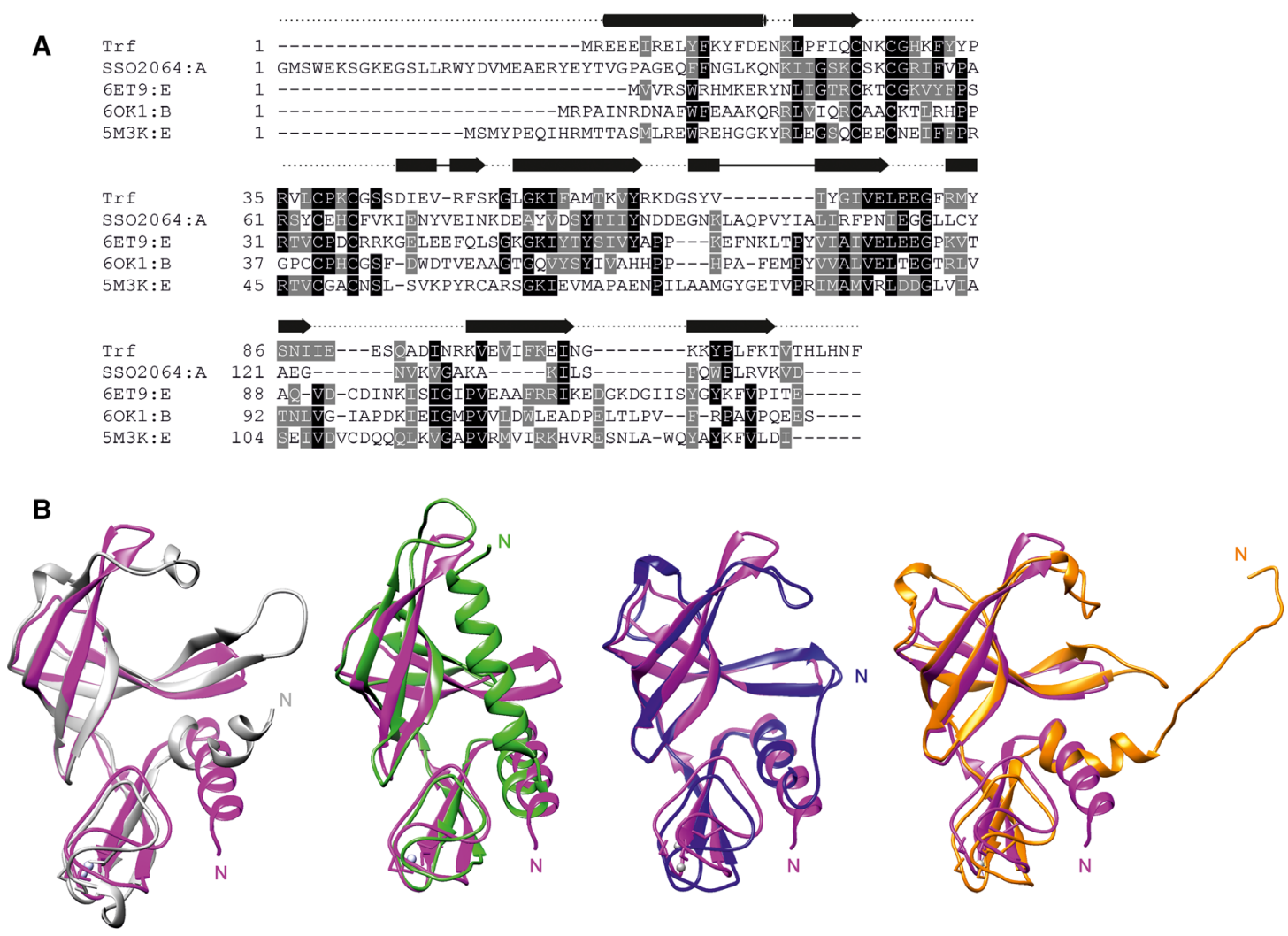

C
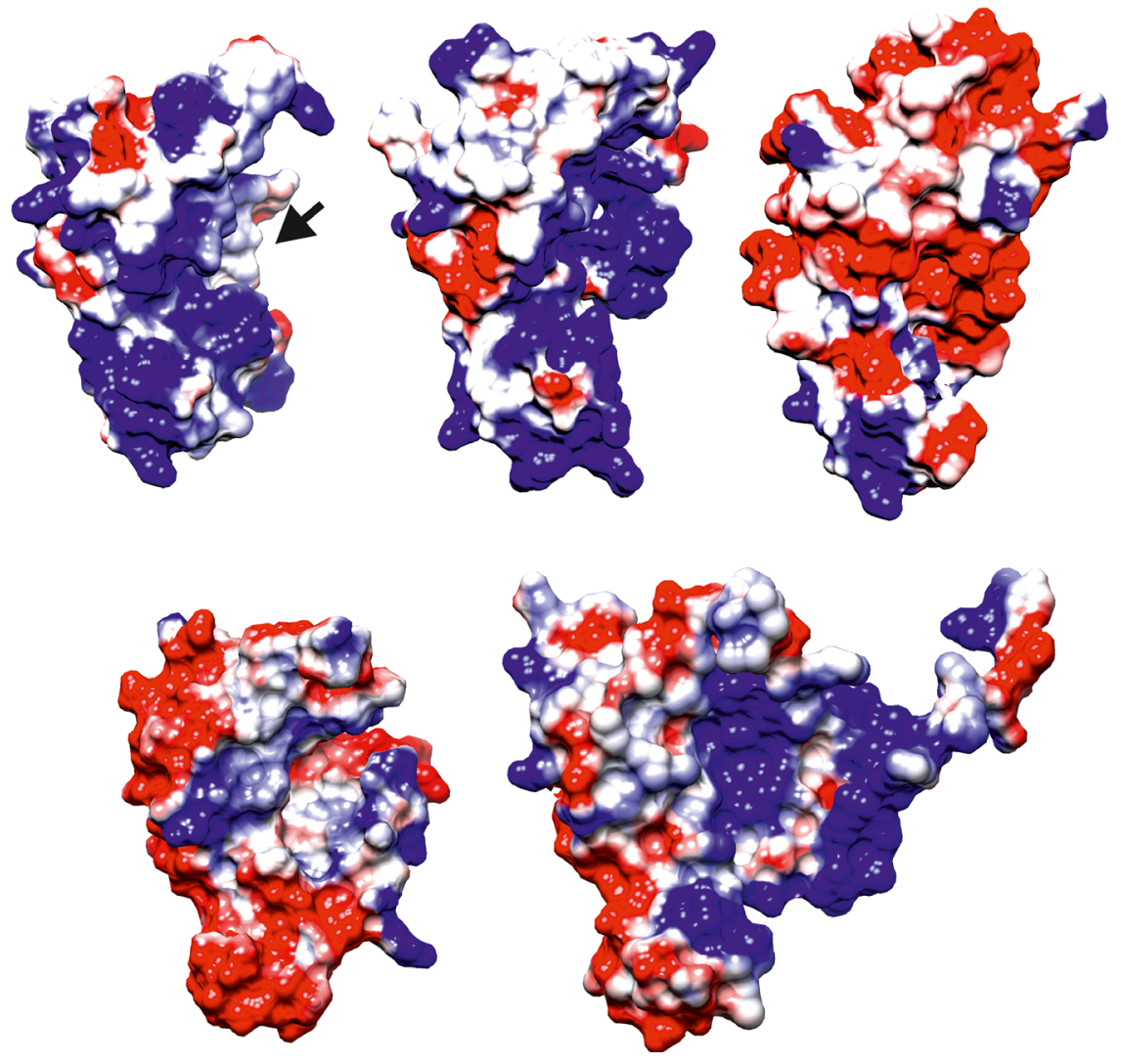
Fig. 3. Sequence comparison and structural similarities between Trf and the other two structurally characterized DUF35 domain-containing proteins. (A) Sequence alignment of Trf, 3irb, 6et9: chain E, 6ok1: chain B, and 5m3k: chain B. Conservation is shown in black (identical residues) and gray (similar residues). The secondary structure of Trf is indicated above the alignment by arrows and cylinders for $\beta$-strands and $\alpha$-helices, respectively. (B) Superimpositions of 6et9: chain E (left, gray), 3irb (second from left, forest green), 6ok1: chain B (second from right, blue), and 5m3k: chain B (right, orange) onto Trf (magenta). (C) Comparison of the electrostatic surface characteristics of Trf (upper row, left), 6et9 chain E (upper row, middle), 3irb (upper row, right), 6ok1: chain B (lower row, left), and 5m3k: chain B (lower row, right). An arrow indicates the position of the positively charged putative RNA binding cleft of Trf.

between $\operatorname{Trf}$ and all other structurally characterized DUF35 proteins is rather low (22\% for Sso $2509,24 \%$ for chain $\mathrm{E}$ of $6 \mathrm{et} 9,22 \%$ for chain $\mathrm{B}$ of $6 \mathrm{ok} 1$, and $19 \%$ for chain B of $5 \mathrm{~m} 3 \mathrm{k}$ ). A sequence alignment of the proteins is shown in Fig. 3A. Importantly, the electrostatic surface potentials of the proteins differ considerably. The N-terminal helical parts of Trf as well as of chain $\mathrm{E}$ of $6 \mathrm{et} 9$ show a positive electrostatic surface potential, whereas the $\mathrm{N}$-terminal helices of Sso2064 feature a negative surface potential due to the presence of an additional long $\mathrm{N}$-terminal $\alpha$-helix in this protein (Fig. 3C).

Furthermore, the parts of both domains forming the putative 'binding cleft' display a positive surface potential in Trf as well as in chain E of 6et9 and chain B of $5 \mathrm{~m} 3 \mathrm{k}$, while Sso2064 and chain B of 6ok 1 show a negative surface potential in this area (Fig. 3C). Thus, in regard to its overall structure and its surface properties Trf is most similar to chain $\mathrm{E}$ of 6et9. Interestingly, in chain E of 6et9 the positively charged 'binding cleft' between the two domains interacts with the thiolase domain of the enzyme complex. For Trf, this binding cleft could be either the interaction surface for aIF $2 \gamma$ or for its target mRNAs. However, since S. solfataricus aIF $2 \gamma$ features only a rather limited area of negative surface potential compared to the thiolase domain of $M$. thermolithotrophicus, a potential mRNA binding surface seems to be more likely. In analogy to the function of chain E of 6et9, Trf might function in order to establish a transient scaffolding state between $\operatorname{Trf}$, aIF $2 \gamma$, and the bound mRNA to promote the release of bound mRNAs from aIF $2 \gamma$.

Overall, our results demonstrate that while sequentially dissimilar DUF35 proteins fold into highly similar structures, they differ considerably in their surface features and can therefore be recruited into a potentially wide variety of biological pathways with general scaffolding and binding functions.

\section{Acknowledgements}

We are grateful to the Paul Scherrer Institute, Villigen, Switzerland, for providing synchrotron radiation beam time at the beamlines PX and PXIII of the Swiss Light
Source (SLS). This work was supported by funding from the European Community's Seventh Framework Programme (FP7/2007-2013) under BioStruct-X (grant agreement No 283570) and from the Deutsche Forschungsgemeinschaft (DFG) through the SFB 902 'Molecular Mechanisms of RNA-based Regulation' (project B10 to JW).

\section{Conflict of interest}

The authors declare no conflict of interest.

\section{Data accessibility}

The structure of $S$. solfataricus $\operatorname{Trf}$ has been deposited in the PDB under accession code 6HTJ.

\section{Author contributions}

MK overexpressed, purified, and crystallized protein; analyzed data; and wrote the manuscript. JPW provided protocols; assisted in purification, crystallization, and data analysis; and discussed the manuscript. BM provided reagents and protocols. UB provided reagents and protocols, conceptualized the project, analyzed data, and discussed the manuscript. DP collected, analyzed diffraction data, and discussed the manuscript. JW conceptualized the project, analyzed data, and wrote the manuscript.

\section{References}

1 de Rosa M, Gambacorta A and Bu'Lock JD (1975) Extremely thermophilic acidophilic bacteria convergent with Sulfolobus acidocaldarius. Microbiology 86, 156-164.

2 La Teana A, Benelli D, Londei P and Bläsi U (2013) Translation initiation in the crenarchaeon Sulfolobus solfataricus: eukaryotic features but bacterial route. Biochem Soc Trans 41, 350-355.

3 Hasenöhrl D, Fabbretti A, Londei P, Gualerzi CO and Bläsi U (2009) Translation initiation complex formation in the crenarchaeon Sulfolobus solfataricus. RNA 15, 2288-2298. 
4 Milon P, Carotti M, Konevega AL, Wintermeyer W, Rodnina MV and Gualerzi CO (2010) The ribosomebound initiation factor 2 recruits initiator tRNA to the 30S initiation complex. EMBO Rep 11, 312-316.

5 Märtens B, Manoharadas S, Hasenöhrl D, Zeichen L and Bläsi U (2013) Back to translation: removal of aIF2 from the $5^{\prime}$-end of mRNAs by translation recovery factor in the crenarchaeon Sulfolobus solfataricus. Nucleic Acids Res 42, 2505-2511.

6 Arkhipova V, Stolboushkina E, Kravchenko O, Kljashtorny V, Gabdulkhakov A, Garber M, Nikonov S, Märtens B, Bläsi U and Nikonov O (2015) Binding of the $5^{\prime}$-triphosphate end of mRNA to the $\gamma$-subunit of translation initiation factor 2 of the crenarchaeon Sulfolobus solfataricus. J Mol Biol 427, 3086-3095.

7 Krishna SS, Aravind L, Bakolitsa C, Caruthers J, Carlton D, Miller MD, Abdubek P, Astakhova T, Axelrod HL, Chiu HJ et al. (2010) The structure of SSO2064, the first representative of Pfam family PF01796, reveals a novel two-domain zinc-ribbon OBfold architecture with a potential acyl-CoA-binding role. Acta Crystallogr Sect F Struct Biol Cryst Commun 66, 1160-1166.

8 Vögeli B, Engilberge S, Girard E, Riobé F, Maury O, Erb TJ, Shima S and Wagner T (2018) Archaeal acetoacetyl-CoA thiolase/HMG-CoA synthase complex channels the intermediate via a fused CoA-binding site. Proc Natl Acad Sci USA 115, 3380-3385.

9 Aggett R, Mallette E, Gilbert SE, Vachon MA, Schroeter KL, Kimber MS and Seah SYK (2019) The steroid side-chain-cleaving aldolase Ltp2-ChsH2 2 DUF35 is a thiolase superfamily member with a radically repurposed active site. J Biol Chem 294, 11934-11943.

10 Pavkov-Keller T, Schmidt NG, Żądło-Dobrowolska A, Kroutil W and Gruber K (2019) Structure and catalytic mechanism of a bacterial Friedel-Crafts acylase. ChemBioChem 20, 88-95.

11 Kabsch W (2010) Xds. Acta Crystallogr D Biol Crystallogr 66, 125-132.

12 Schneider TR and Sheldrick GM (2002) Substructure solution with SHELXD. Acta Crystallogr D Biol Crystallogr 58, 1772-1779.

13 Sheldrick GM (2002) Macromolecular phasing with SHELXE. Z Kristallogr Cryst Mater 217, 644-650.

14 Sheldrick GM (2010) Experimental phasing with SHELXC/D/E: combining chain tracing with density modification. Acta Crystallogr D Biol Crystallogr 66, 479-485.
15 Terwilliger TC (2003) Automated main-chain model building by template matching and iterative fragment extension. Acta Crystallogr D Biol Crystallogr 59, 38-44.

16 Emsley P and Cowtan K (2004) Coot: model-building tools for molecular graphics. Acta Crystallogr D Biol Crystallogr 60, 2126-2132.

17 Adams PD, Afonine PV, Bunkóczi G, Chen VB, Davis IW, Echols N, Headd JJ, Hung LW, Kapral GJ, Grosse-Kunstleve RW et al. (2010) PHENIX: a comprehensive Python-based system for macromolecular structure solution. Acta Crystallogr D Biol Crystallogr 66, 213-221.

18 Chen VB, Arendall WB 3rd, Headd JJ, Keedy DA, Immormino RM, Kapral GJ, Murray LW, Richardson JS and Richardson DC (2010) MolProbity: all-atom structure validation for macromolecular crystallography. Acta Crystallogr D Biol Crystallogr 66, 12-21.

19 Pettersen EF, Goddard TD, Huang CC, Couch GS, Greenblatt DM, Meng EC and Ferrin TE (2004) UCSF Chimera - a visualization system for exploratory research and analysis. J Comput Chem 25, 1605-1612.

20 Holm L, Kääriäinen S, Rosenström P and Schenkel A (2008) Searching protein structure databases with DaliLite vol 3. Bioinformatics 24, 2780-2781.

21 Krissinel E and Henrick K (2004) Secondary-structure matching (SSM), a new tool for fast protein structure alignment in three dimensions. Acta Crystallogr D Biol Crystallogrv 60, 2256-2268.

22 Hellmich UA, Weis BL, Lioutikov A, Wurm JP, Kaiser M, Christ NA, Hantke K, Kötter P, Entian KD, Schleiff E et al. (2013) Essential ribosome assembly factor Fap7 regulates a hierarchy of RNA-protein interactions during small ribosomal subunit biogenesis. Proc Natl Acad Sci USA 110, 15253-15258.

23 Murzin AG (1993) OB (oligonucleotide/oligosaccharide binding)-fold: common structural and functional solution for non-homologous sequences. EMBO J 12, 861-867.

24 Krissinel E and Henrick K (2007) Inference of macromolecular assemblies from crystalline state. $J \mathrm{Mol}$ Biol 372, 774-797.

25 Theobald DL, Mitton-Fry RM and Wuttke DS (2003) Nucleic acid recognition by OB-fold proteins. Annu Rev Biophys 32, 115-133. 